\title{
Erratum to: The Contribution of Cdc2 in Rotenone-Induced G2/M Arrest and Caspase-3-Dependent Apoptosis
}

\author{
Hongcai Wang • Zhentao Zhang • Jinsha Huang • \\ Ping Zhang $•$ Nian Xiong $•$ Tao Wang
}

Published online: 4 February 2014

(C) Springer Science+Business Media New York 2014

\section{Erratum to: J Mol Neurosci (2013) \\ DOI 10.1007/s12031-013-0185-3}

The original version of this article unfortunately contained mistakes. The changes are emphasized in bold as follows:

Page 2, "Reagents" section:

"Rabbit anti-CDK2 (\#2546), rabbit anti-p-cdc2 (Thr161) antibody (\#9114), rabbit anti-CDK5 antibody (\#2506), rabbit anti-cleaved caspase-3 (Asp175)(5A1E) antibody (\#9664) and rabbit polyclonal anti-caspase-9 antibody (\#9502) were obtained from Cell Signaling Technology at a dilution of 1:200-1:1,000.”

\section{Should read:}

"Rabbit CDK2 (\#2546), rabbit p-cdc2 (Thr161) (\#9114), rabbit CDK5 (\#2506), rabbit cleaved caspase-3 (Asp175)(5A1E) (\#9664), mouse Cdc2 (POH1) (\#9116) and rabbit polyclonal caspase-9 (\#9502) antibodies were obtained from Cell Signaling Technology at a dilution of 1:200-1:1,000."

Page 2, "Hoechst Staining and Confocal Fluorescence Microscopy" section:

"After washing with PBS, the cells were incubated with FITCconjugated goat anti-rabbit IgG and $\mathrm{Cy} 3$-conjugated goat anti-

The online version of the original article can be found at http://dx.doi.org/ 10.1007/s12031-013-0185-3.

H. Wang $\cdot$ J. Huang $\cdot$ P. Zhang $\cdot$ N. Xiong $\cdot$ T. Wang $(\bowtie)$

Department of Neurology, Union Hospital, Tongji Medical College,

Huazhong University of Science and Technology,

1277 Jie Fang Road, Wuhan 430022, Hubei, China

e-mail: wangtaowh@hust.edu.cn

\section{Z. Zhang}

Department of Neurology, Renmin Hospital of Wuhan University,

Wuhan 430060, China mouse IgG $37^{\circ} \mathrm{C}$ for $1 \mathrm{~h}$ and nuclear counterstained by the blue fluorescence of Hoechst $(5.0 \mu \mathrm{g} / \mathrm{ml})$ for $10 \mathrm{~min} . "$

Should read:

"After washing with PBS, the cells were incubated with FITCconjugated goat anti-mouse IgG and $\mathrm{Cy} 3$-conjugated goat anti-rabbit IgG $37^{\circ} \mathrm{C}$ for $1 \mathrm{~h}$ and nuclear counterstained by the blue fluorescence of Hoechst $(5.0 \mu \mathrm{g} / \mathrm{ml})$ for $10 \mathrm{~min} . "$

Page 3, "Caspase-3 Was Activated Both in Cytoplasm and Nucleus Accompanied by Cell Cycle G2/M Arrest" section, third paragraph:

"The proportion of cells in G2/M phase was elevated in a time-dependent manner until 36 h (Fig. 1b)."

\section{Should read:}

"The proportion of cells in G2/M phase was elevated in a time-dependent manner until $36 \mathrm{~h}$ (Fig. 1d)."

Page 4, "The Decreased Activity and the Increased Aggregation of Cdc2 Occurred After Rotenone Treatment" section, first paragraph:

"In selective enlargement, dot accumulation of Cdc2 was easily found in the cytoplasm (Fig. 2b, arrow)."

\section{Should read:}

"In selective enlargement, dot accumulation of Cdc2 was easily found in the cytoplasm (Fig. 2a, arrow)."

Page 7, "The siRNA-Mediated Cdc2 Knockdown Decreased Cleaved Caspase-3/9 Expression and Protected Against Rotenone-Induced Cell Apoptosis" section, third paragraph:

"We found that the expression of cleaved caspase-9 and -3 were elevated significantly after a 12 -h exposure to rotenone and further elevation at $36 \mathrm{~h}$ (Fig. 5)." 
Should read:

"We found that the expression of cleaved caspase-9 and -3 were elevated significantly after a 12 -h exposure to rotenone and further elevation at $36 \mathrm{~h}$ (Fig. 5c)."

Page 9, "Discussion" section, fifth paragraph:

"Previous studies suggest that in neuron cell cycle reentry, the prolonged G2 stage might not undergo cell division but instead induce apoptosis (Cabeza-Arvelaiz et al. 2012)."

Should read:

"Previous studies suggest that in neuron cell cycle reentry, the prolonged G2 stage might not undergo cell division but instead induce apoptosis (Cabeza-Arvelaiz and Schiestl 2012)." 